\title{
On the Simulation of $\mathrm{M}_{2}$ Tide in the Bohai, Yellow, and East China Seas with TOPEX/Poseidon Altimeter Data
}

\author{
Jicai Zhang ${ }^{1}$ and Xianqing $\mathrm{Lu}^{1, *}$ \\ 1 Laboratory of Physical Oceanography, Ocean University of China, Qingdao 266003, China
}

Received 15 November 2006, accepted 23 June 2007

\begin{abstract}
The $\mathrm{M}_{2}$ tide in the Bohai, Yellow, and East China Seas (BYECS) is simulated by assimilating the TOPEX/Poseidon altimetry data with the adjoint method. The bottom friction coefficients (BFC) are supposed to be spatially varying. In this paper, the BFC at some grid points are chosen as the independent BFC and the BFC at the other grid points can be obtained through linear interpolation with these independent BFC. The independent BFC are selected uniformly over each $1^{\circ} \times 1^{\circ}$ area and 120 independent BFC are obtained. Twin and practical experiments are carried out to discuss the spatially varying BFC. The results of twin experiments demonstrate that the adjoint method has a strong ability of inverting the prescribed BFC distributions combined with the spatially varying BFC. The prescribed BFC distributions are all successfully inverted. In practical experiments, the simulation precision has been highly increased by optimizing the spatially varying independent BFC. Three amphidromic points and a degenerate one appear in the cotidal charts of $\mathrm{M}_{2}$ tide and the results coincide with the observed $\mathrm{M}_{2}$ tide in BYECS fairly well.
\end{abstract}

Key words: TOPEX/Poseidon, Spatially varying parameters, Tidal model, Adjoint method

Citation: Zhang, J., and X. Lu, 2008: On the simulation of $M_{2}$ tide in the Bohai, Yellow, and East China Seas with TOPEX/Poseidon altimeter data. Terr. Atmos. Ocean. Sci., 19, 173-181, doi: 10.3319/TAO.2008.19.1-2.173(SA)

\section{INTRODUCTION}

Many global tidal models have been established since the launch of TOPEX/Poseidon (T/P) satellite (Egbert et al. 1994; Le Provost et al. 1994; Mazzega and Berge 1994; Anderson 1995; Kantha 1995). People's understanding about the tide has been greatly improved due to the large amount of sea level elevation data provided by T/P. The first application of T/P altimeter data in seas adjacent to China was carried out by Mazzega and Berge (1994) to derive ocean tides in the East Asian Marginal seas and the data covered only about 7 months. Yanagi et al. (1997) then used T/P data covering about 3 years to construct cotidal charts for the Yellow and East China Seas and the results showed significant improvement over Mazzega and Berge's work. Teague et al. (2000) obtained the tides in the Bohai and Yellow Seas derived from 5 years of T/P data. Using FES94.1, Lefevre et al. (2000) simulated the tide in the Yellow and East China Seas and discussed the problem how to improve a global ocean tide model at a regional scale. In the work of Fang et al. (2004), harmonic analysis of 10 years of T/P along-track altimetry was performed to derive the semidiurnal, diurnal, long-period and quarter-diurnal tides in the Bohai, Yellow, and East China Seas (BYECS).

$\mathrm{T} / \mathrm{P}$ altimetry has reopened the problem of how tidal dissipation is to be allocated (Munk 1997). The bottom friction is closely related to the ocean topography and plays an important role in the tidal phenomenon. For example, tidal dissipation models are usually based on a frictional bottom boundary layer in which the work done by the bottom friction is proportional to the friction coefficient and the velocity cubed (Munk 1997). Therefore, it is necessary to depict the bottom friction effect clearly. In tidal models the bottom friction effect is parametrized by the bottom friction coefficients (BFC). So far, the following methods have been employed to deal with the BFC. First, the BFC has been

\footnotetext{
* Corresponding author

E-mail:xqinglv@mail.ouc.edu.cn
} 
taken as a constant over the whole computing area (Lefevre et al. 2000; Egbert et al. 2004). For example, in the work of Lefevre et al. (2000), 0.0015 is taken as the value of BFC. Egbert et al. (2004) used a constant BFC (0.0025) to simulate the global semidiurnal tide in the present day and in the last glacial maximum. Second, the computing domain is divided into several subdomains and a different BFC is used in each subdomain (Zhao et al. 1993; Le Provost et al. 1994; Kang et al. 1998; He et al. 2004). Zhao et al. (1993) simulated the tide in BYECS and the BFC are taken as 0.001 at the west of the line from $\left(25^{\circ} 15^{\prime} \mathrm{N}, 120^{\circ} 45^{\prime} \mathrm{E}\right)$ to $\left(40^{\circ} 00^{\prime} \mathrm{N}\right.$, $\left.124^{\circ} 15^{\prime} \mathrm{E}\right), 0.0035$ in the Korean Strait and the Strait of Tsushima, and 0.0016 in the other areas. For the global tidal simulation of FES94.1, Le Provost et al. (1994) took a friction coefficient of 0.003 in the pelagic areas of the ocean surface and 0.0025 in the shallow water ones. Third, the BFC are supposed to be spatially varying (Das and Lardner 1991, 1992; Ullman and Wilson 1998; Heemink et al. 2002). Das and Lardner $(1991,1992)$ inverted the position dependent drag coefficients and water depth by assimilating sea level observations at a number of tidal stations. Ullman and Wilson (1998) studied the spatial variability of the BFC by assimilating the ADCP data into a tidal model of the lower Hudson estuary with the adjoint method. Heemink et al. (2002) developed an inverse 3D shallow water flow model in which the spatially varying friction parameters were estimated and the independent BFC were selected by analyzing the parameter gradient per grid point. The first two methods have been widely used in the tidal simulations, but the accuracy is relatively low. Additionally, the calibration of BFC depends on experience, which leads to low efficiency. The third method can be realized only if the adjoint method is employed. Reasonable simulation results can be obtained by optimizing the spatially varying $\mathrm{BFC}$ and the calibration can become an automatic process done by computer. The spatially varying BFC will be discussed with an adjoint tidal model in this paper. The independent $\mathrm{BFC}$ are selected uniformly over each $1^{\circ} \times 1^{\circ}$ area and 120 independent BFC are obtained.

Based on the simulation of $\mathrm{M}_{2}$ tide in BYECS, a series of experiments are carried out to study the spatially varying BFC by assimilating the T/P altimeter data. In this study, the open boundary conditions are fixed. In twin experiments, the prescribed BFC distributions are inverted successfully with the combination of spatially varying BFC and the adjoint method. In practical experiments, the precision of simulation is efficiently increased by optimizing the spatially varying independent $\mathrm{BFC}$ and the simulation results coincide with the observed $\mathrm{M}_{2}$ tide in BYECS fairly well.

The paper is organized as follows. The adjoint tidal model is presented in section 2. In section 3, numerical experiments including twin and practical ones are carried out and the results are analyzed. The summary of section 4 completes the paper.

\section{NUMERICAL ADJOINT TIDAL MODEL}

\subsection{Model Settings}

The forward equations, adjoint equations and their numerical schemes are all the same as those of Lu and Zhang (2006). The computing area in the present study is BYECS $\left(117.5-131^{\circ} \mathrm{E}, 24-41^{\circ} \mathrm{N}\right.$, Fig. 1). From Fig. 1, one can find that the water is shallow in the Bohai and Yellow Seas and deep in the East China Sea. The deepest water appears in and around the Okinawa Trough. The space resolution is $1 / 6^{\circ} \times 1 / 6^{\circ}$. The open boundaries, the position of tidal gauge stations and the T/P satellite tracks are shown in Fig. 2. The satellite tracks contain 6 descending tracks $(036,062,138$, 164, 214, and 240) and 6 ascending tracks $(051,077,127$, 153, 203, and 229). The open boundaries are set along the Taiwan Strait, the first island chains, the Korea Strait and the Tsushima Strait. The angular frequency of $\mathrm{M}_{2}$ tide is $1.405189025 \times 10^{-4} \mathrm{~s}^{-1}$ and the time step is $62.103 \mathrm{~s}(1 / 720$ of the period of $\mathrm{M}_{2}$ tide).

Closed boundary conditions for our model are zero flow normal to the coast. That is $\overrightarrow{\boldsymbol{u}} \cdot \overrightarrow{\boldsymbol{n}}=\mathbf{0}$, for the grid points at closed boundary, where $\overrightarrow{\boldsymbol{n}}$ is the outward unit vector and $\overrightarrow{\boldsymbol{u}}=(\boldsymbol{u}, \boldsymbol{v})$ is the velocity vector. In the present study, Beijing standard time (referring to the meridian of $120^{\circ} \mathrm{E}$ ) will be adopted throughout.

Along the open boundaries, water elevation of $\mathrm{M}_{2}$ tide at the $j$ th time step is given as $\zeta_{m_{b} n_{l}}^{j}=a_{0, l}+\left[a_{l} \cos (\omega j \Delta t)+\right.$ $\left.b_{l} \sin (\omega j \Delta t)\right]$, where $\left(m_{l}, n_{l}\right)$ stands for the grid points at the open boundaries, $\omega$ denotes the frequency of $\mathrm{M}_{2}$ constituent, and $a_{l}, b_{l}$ are the Fourier coefficients. In all the experiments,

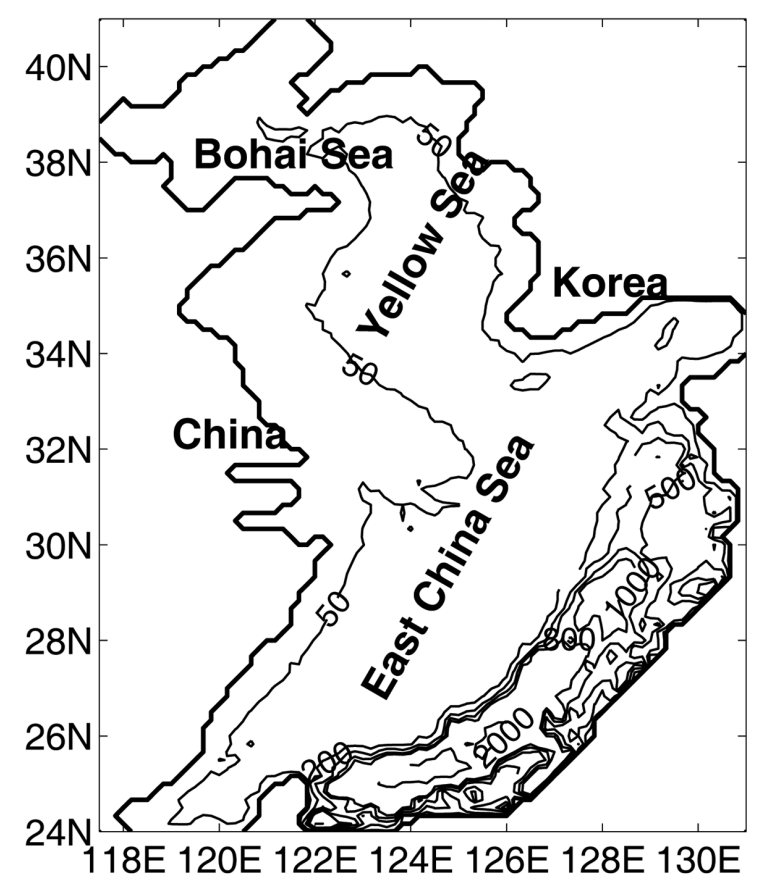

Fig. 1. Bathymetric map of the Bohai Sea, the Yellow Sea and the East China Sea. 
the open boundary conditions of water elevation which are obtained by assimilating $\mathrm{T} / \mathrm{P}$ data with adjoint method are fixed (He et al. 2004).

\subsection{Data}

$\mathrm{T} / \mathrm{P}$ data are assimilated into the numerical model in the present study, and observations from tidal gauge stations are used as an independent check of the model fidelity in the practical experiments. The tidal gauge data and the T/P process are the same as He et al. (2004).

\subsection{Selection and Optimization of Independent BFC}

In this paper, the independent $\mathrm{BFC}$ are selected uniformly over each $1^{\circ} \times 1^{\circ}$ area and 120 independent BFC are obtained (Fig. 3). Assuming $b_{m, n}$ is the independent BFC and $k_{j, k}$ the result of linear interpolation with $b_{m, n}$ yields (Das and Lardner 1991, 1992; Ullman and Wilson 1998):

$k_{j, k}=\sum_{m, n} \phi_{j, k, m, n} \times b_{m, n}$

where $\phi_{j, k, m, n}$ is the coefficient of linear interpolation $\left(\phi_{j, k, m, n}=W_{j, k, m, n} / \sum_{m, n} W_{j, k, m, n}\right), W_{j, k, m, n}$ is the weighted coefficient $\left[W_{j, k, m, n}=\left(R^{2}-r_{j, k, m, n}^{2}\right) /\left(R^{2}+r_{j, k, m, n}^{2}\right)\right]$ of the Cressman form (Cressman 1959), $r_{j, k, m, n}$ is the distance from grid point $(j, k)$ to $(m, n)$, and $R$ is the influence radius taken as $2^{\circ}$ here. The gradients of the Lagrangian function with respect to the independent BFC are given by:

$$
\begin{aligned}
& \frac{\partial L}{\partial b_{m, n}}= \\
& \quad \sum_{i, j, k}\left\{\frac{\phi_{j, k, m, n}+\phi_{j+1, k, m, n}}{2} \times \frac{u_{a j, k}^{i+1} r_{j, k}^{i}\left[\alpha u_{j, k}^{i+1}+(1-\alpha) u_{j, k}^{i}\right]}{h_{j+1 / 2, k}+\xi_{j+1 / 2, k}^{i}}\right\} \\
& +\sum_{i, j, k}\left\{\frac{\phi_{j, k, m, n}+\phi_{j, k+1, m, n}}{2} \times \frac{v_{a j, k}^{i+1} s_{j, k}^{i}\left[\alpha v_{j, k}^{i+1}+(1-\alpha) v_{j, k}^{i}\right]}{h_{j+1 / 2, k}+\xi_{j+1 / 2, k}^{i}}\right\}
\end{aligned}
$$

where $i$ is the index of time. Because the cost function decreases along the opposite direction of the gradient, we can obtain the correction of the independent BFC as:

$$
K_{c}\left(b_{m, n}-\hat{b}_{m, n}\right)+\frac{\partial L}{\partial b_{m, n}} /\left\|\frac{\partial L}{\partial b}\right\|_{2}=0
$$

i.e.,

$$
b_{m, n}=\hat{b}_{m, n}-\frac{1}{K_{c}} \frac{\partial L}{\partial b_{m, n}} /\left\|\frac{\partial L}{\partial b}\right\|_{2}=0
$$

where

$$
\left\|\frac{\partial L}{\partial b}\right\|_{2}=\left(\sum_{m, n} \frac{\partial L}{\partial b_{m, n}}\right)^{2},
$$

where $\hat{b}_{m, n}$ and $b_{m, n}$ are prior and optimized values of independent $\mathrm{BFC}$, respectively, $K_{c}$ is a constant. In our model, $\partial L / \partial b_{m, n}$, which is the gradient of the Lagrangian function with respect to the independent BFC, is normalized first, which means that mainly the direction of the gradient is used when the optimization is performed. A small positive value is assigned to $1 / K_{c}$ in order to ensure the cost function can decrease continuously without fluctuations. In our model, the initial value is set to 0.002 and $1 / K_{c}$ is taken as $10^{-4}$, i.e., $K_{c}=10^{4}$ which is obtained through a trial and error procedure.

\section{NUMERICAL EXPERIMENTS AND RESULT ANALYSIS}

\subsection{Twin Experiments}

In the twin experiments, BFC distributions are prescribed and then the strategy described above is used to invert the given distributions using the adjoint method. The 'observations' are produced by the forward tidal model and recorded at the position of $\mathrm{T} / \mathrm{P}$ altimeter data. The procedure of inversion is given as follows.

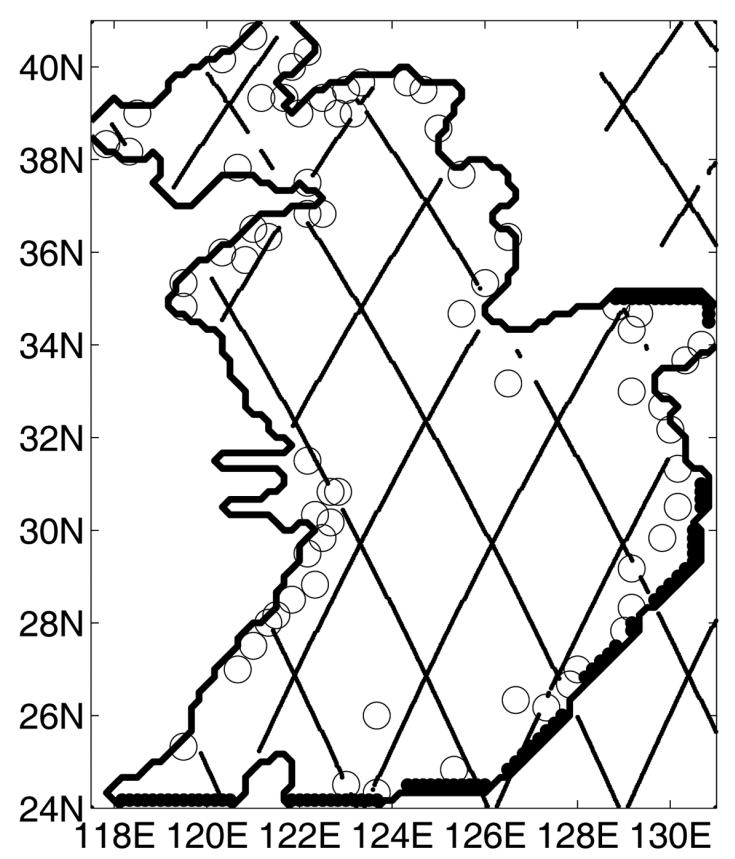

Fig. 2. Position of T/P satellite tracks, tide gauge stations, and open boundaries. The dots indicate the points at the open boundaries and the open circles indicate the tidal gauge stations. The bold lines are the $\mathrm{T} / \mathrm{P}$ satellite tracks. 


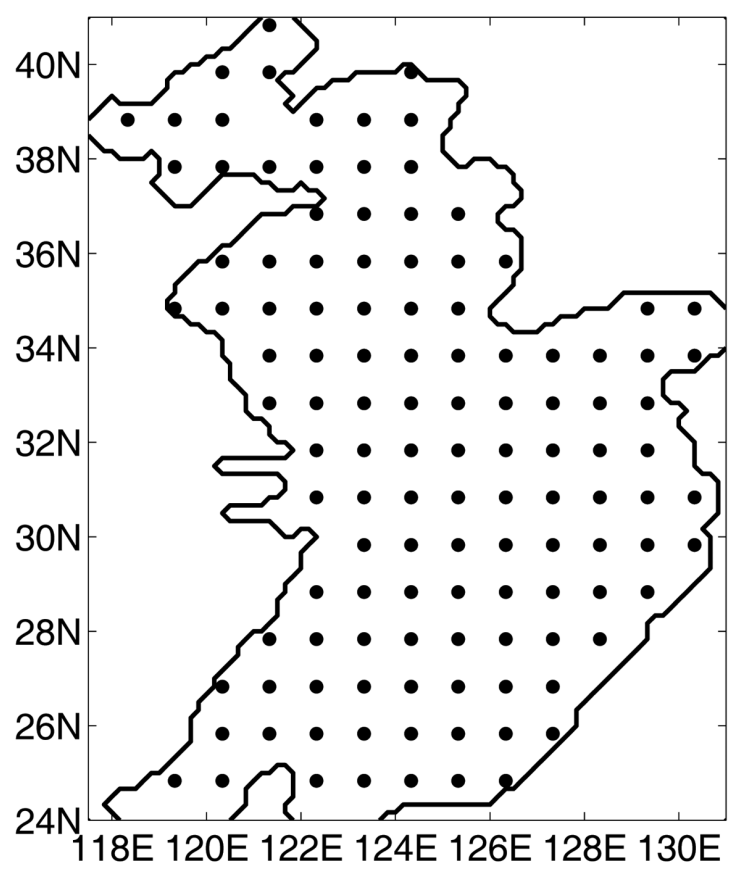

Fig. 3. Distribution of independent BFC.

First, an initial value (0.002) is assigned to the independent $\mathrm{BFC}$ and the $\mathrm{BFC}$ at each grid point can be obtained through linear interpolation. Then the forward simulation is performed and the difference of water elevation between simulated values and the 'observations' serves as the external force of adjoint equations. The optimized independent BFC can be obtained through the backward integration of the adjoint equations. The inverse integral time of the adjoint equation is equal to a period of $\mathrm{M}_{2}$ tide. With the procedure above repeated, the independent $\mathrm{BFC}$ will be optimized continuously and the difference of water elevation between simulated values and 'observations' will be diminished. Meanwhile, the difference between the prescribed and the inversed BFC will also be decreased. The number of iteration steps that is needed to invert the prescribed distributions successfully has relation to $K_{c}$ and the initial value. In our study, we monitor the convergence by examining the cost function and terminate the iteration when the change becomes very small. For $K_{c}=10^{4}$ and the initial value $0.002,150$ iteration steps are appropriate because both the cost function and the differences will be decreased very slowly at this step. Therefore, the optimization is terminated at the $150^{\text {th }}$ step.

3 types of surface distributions of BFC have been inverted to testify the validity of the method. These surfaces contain conical surface (linear surface), revolution parabolic surface (quadric surface) and revolution normal surface (hyper surface). Because each surface has two convex directions, we did 6 twin experiments including the inversion of conical surfaces (case 1 and case 2), revolution parabolic surfaces (case 3 and case 4 ) and revolution normal surfaces (case 5 and case 6). According to the previous works on $\mathrm{BFC}$, the prescribed values are between 0.001 and 0.003 .

Table 1 gives the differences of water elevation between simulated values and 'observations' before and after assimilation. Table 2 shows the average absolute differences, the average relative differences and the correlation coefficient between inverted $\mathrm{BFC}$ and prescribed $\mathrm{BFC}$ before and after assimilation. The prescribed and the inverted BFC of cases 1 - 6 are plotted in Figs. 4 - 9, respectively.

From Figs. 4 - 9 one can find that the prescribed BFC distributions have been successfully inverted in the twin experiments. Tables 1 and 2 also imply this conclusion. In Table 1, the absolute mean differences of amplitude and phase-lag of water elevation between simulated values and 'observations' decreased greatly after assimilation. So are the average absolute differences and the average relative differences between inverted BFC and prescribed BFC in Table 2. The correlation coefficients are all larger than 0.80 which demonstrates the inversion ability of the adjoint

Table 1. The absolute mean differences of amplitude and phase-lag of water elevation between simulated values and 'observations'

\begin{tabular}{ccccc}
\hline \multirow{2}{*}{ EXP. } & \multicolumn{2}{c}{ Before Assimilation } & \multicolumn{2}{c}{ After Assimilation } \\
\cline { 2 - 5 } & Amplitude $(\mathbf{c m})$ & Phase_lag $\left(^{\circ}\right)$ & Amplitude $(\mathbf{c m})$ & Phase_lag $\left(^{\circ}\right)$ \\
\hline Case 1 & 2.16 & 1.22 & 0.04 & 0.03 \\
Case 2 & 2.58 & 1.36 & 0.12 & 0.05 \\
Case 3 & 3.87 & 1.58 & 0.07 & 0.05 \\
Case 4 & 5.16 & 2.08 & 0.24 & 0.09 \\
Case 5 & 3.28 & 1.50 & 0.05 & 0.06 \\
Case 6 & 6.03 & 2.33 & 0.27 & 0.13 \\
\hline
\end{tabular}


Table 2. The average absolute differences (AAD), the average relative differences (ARD) and the correlation coefficient between inverted BFC and prescribed BFC before assimilation (BA) and after assimilation (AA).

\begin{tabular}{cccccc}
\hline \multirow{2}{*}{ EXP. } & \multicolumn{2}{c}{ AAD } & \multicolumn{2}{c}{ ARD } & $\begin{array}{c}\text { Correlation } \\
\text { Coefficient }\end{array}$ \\
\cline { 2 - 6 } Case 1 & BA & AA & BA & AA & 0.95 \\
Case 2 & $1.46 \mathrm{E}-4$ & $8.05 \mathrm{E}-05$ & $15.5 \%$ & $3.9 \%$ & 0.94 \\
Case 3 & $1.26 \mathrm{E}-3$ & $1.18 \mathrm{E}-04$ & $21.6 \%$ & $4.3 \%$ & 0.91 \\
Case 4 & $1.33 \mathrm{E}-3$ & $1.73 \mathrm{E}-04$ & $46.1 \%$ & $11.9 \%$ & 0.84 \\
Case 5 & $1.06 \mathrm{E}-3$ & $9.26 \mathrm{E}-05$ & $19.1 \%$ & $3.7 \%$ & 0.94 \\
Case 6 & $1.34 \mathrm{E}-3$ & $2.40 \mathrm{E}-04$ & $58.8 \%$ & $18.2 \%$ & 0.80 \\
\hline
\end{tabular}

(a)

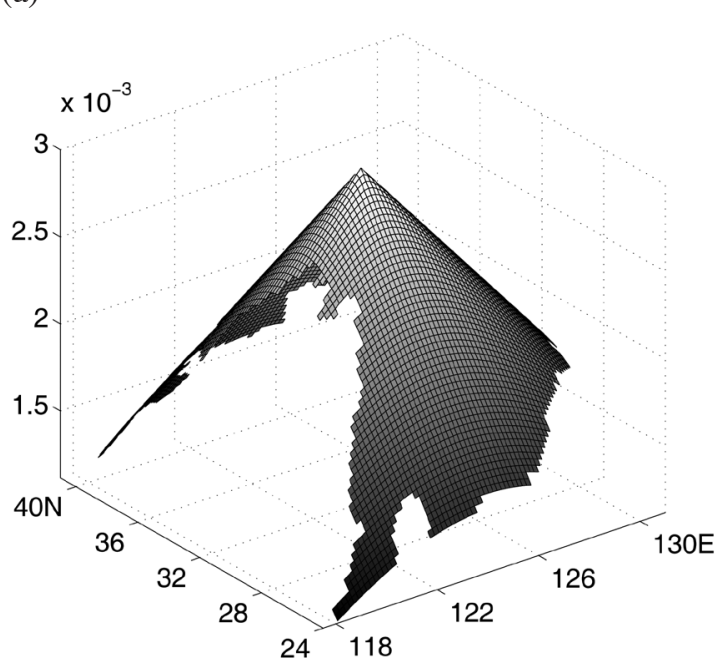

(b)

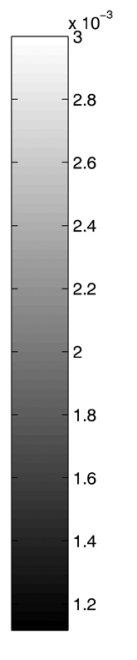

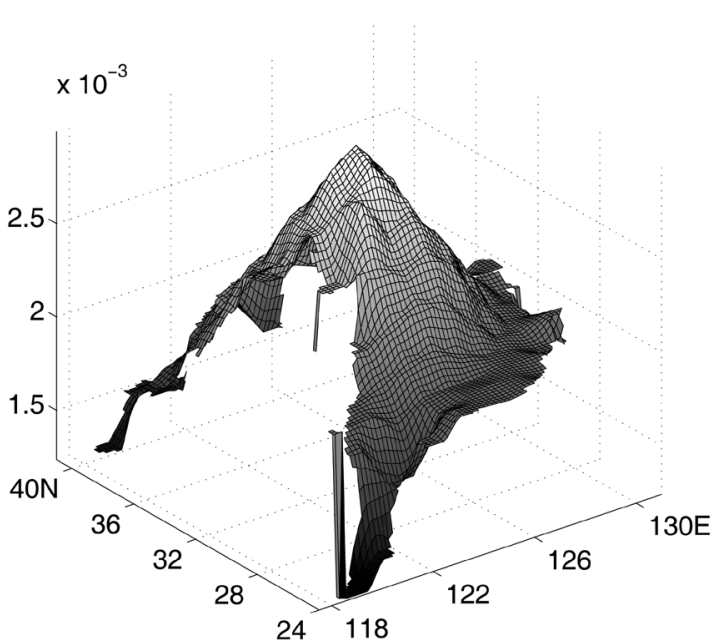

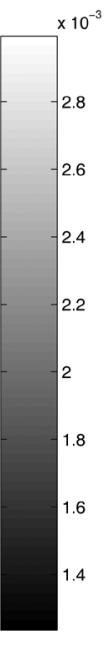

Fig. 4. The spatial distribution of prescribed (a) and inverted (b) BFC of case 1.

(a)

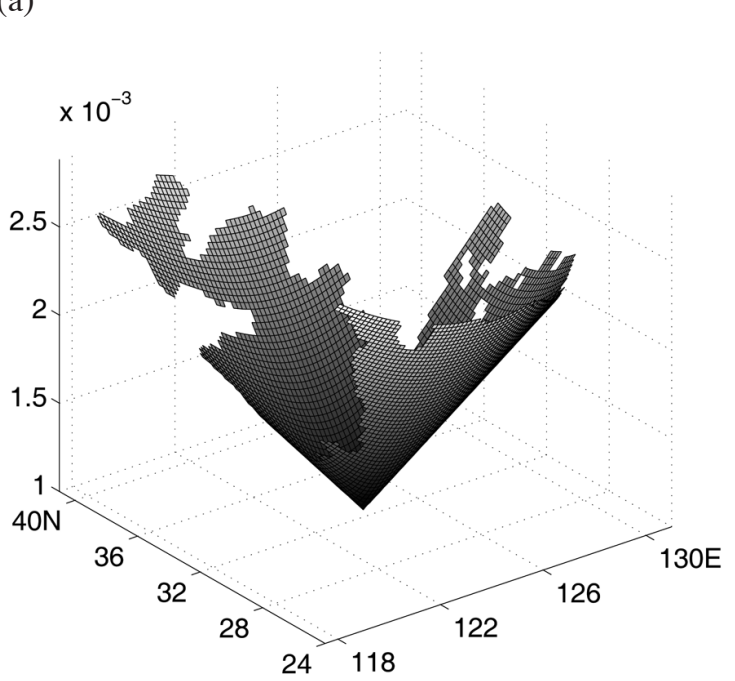

(b)

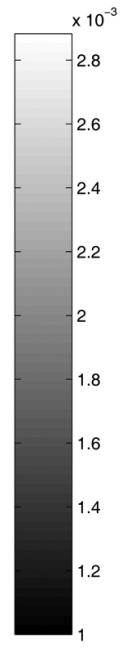

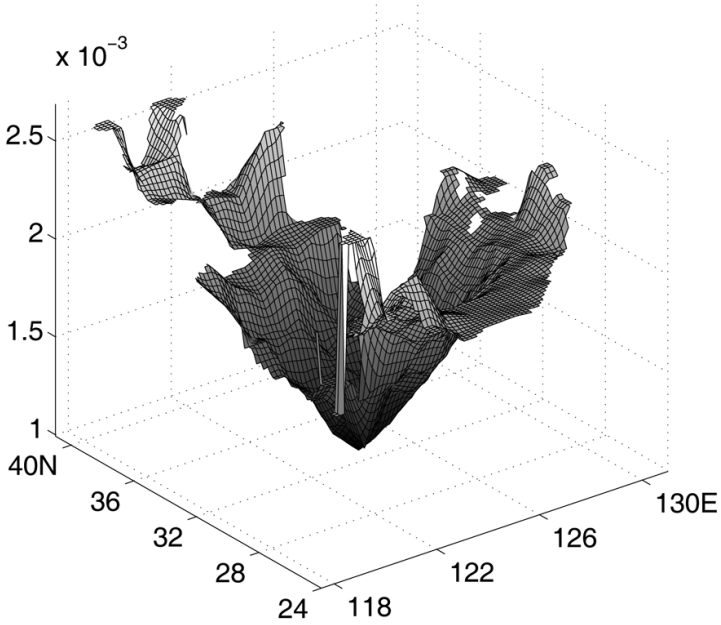

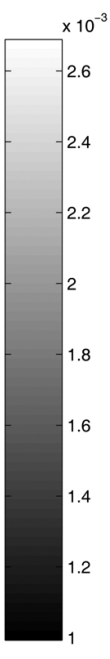

Fig. 5. Same as Fig. 4, but for case 2. 
(a)

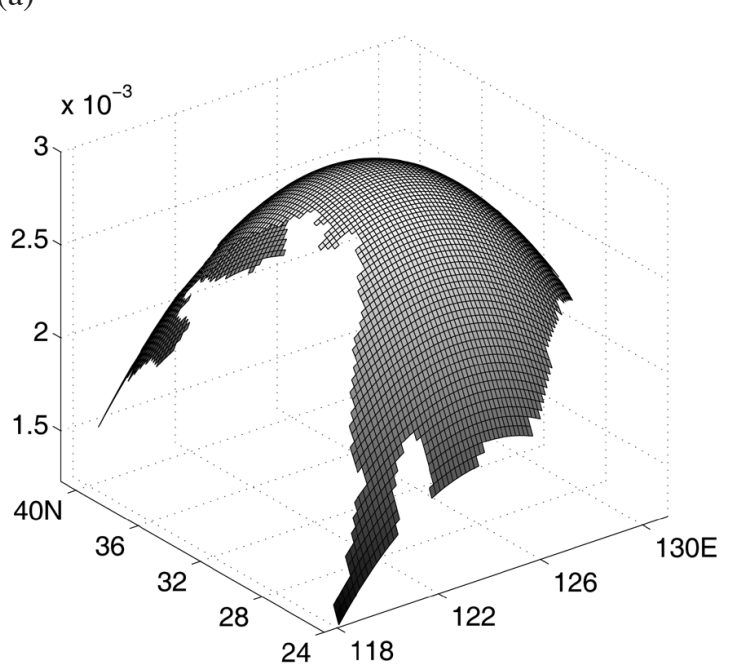

(b)

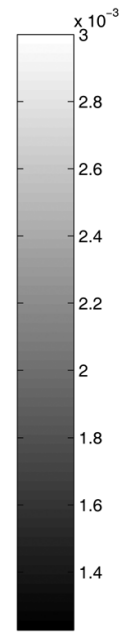

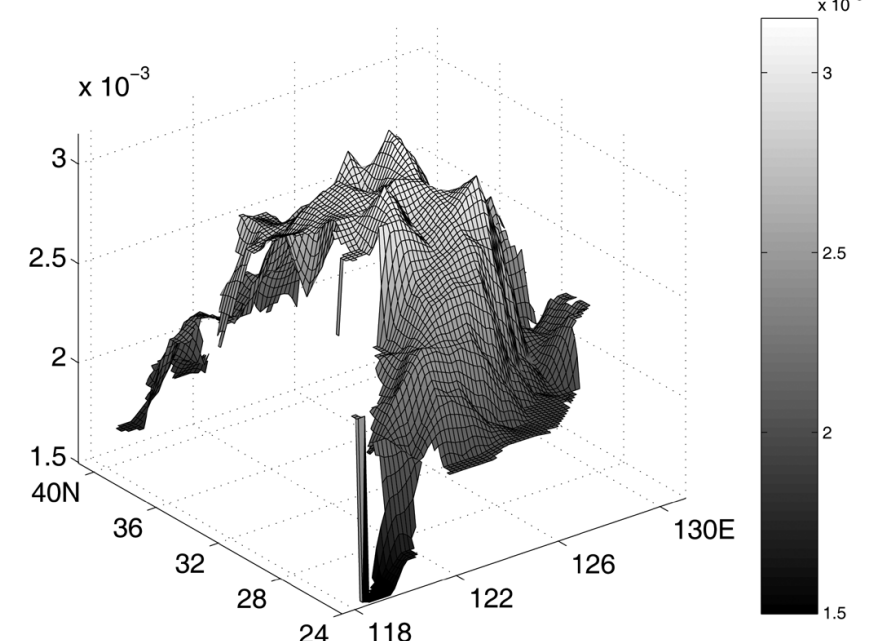

Fig. 6. Same as Fig. 4, but for case 3.

(a)

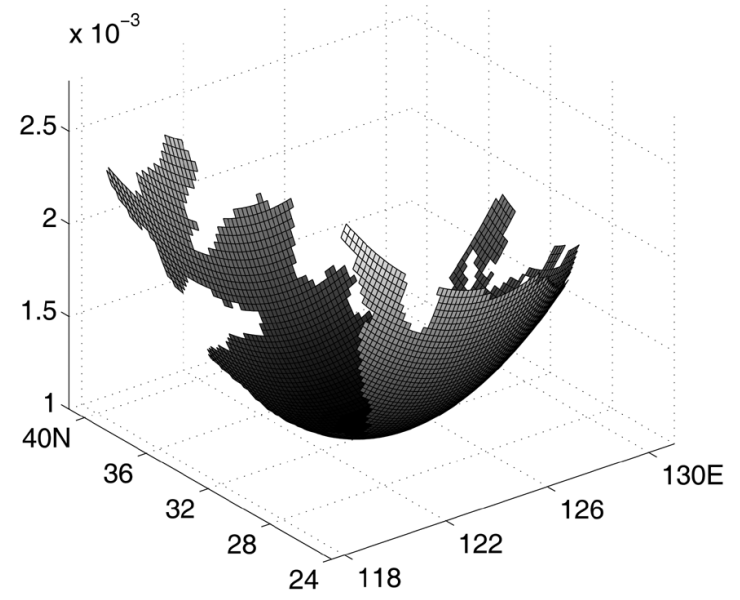

(b)

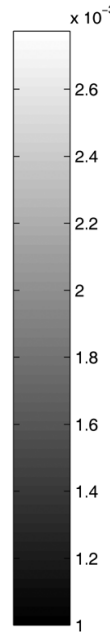

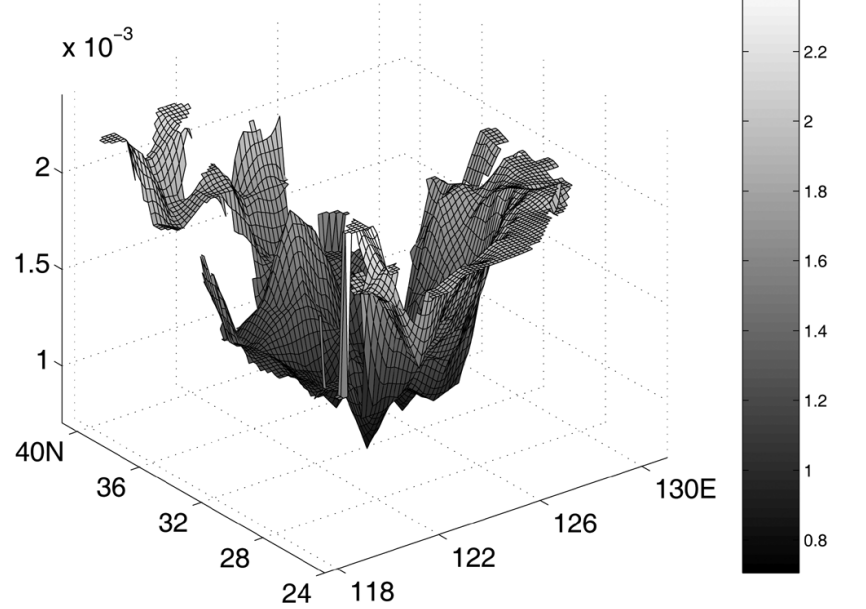

Fig. 7. Same as Fig. 4, but for case 4.

(a)

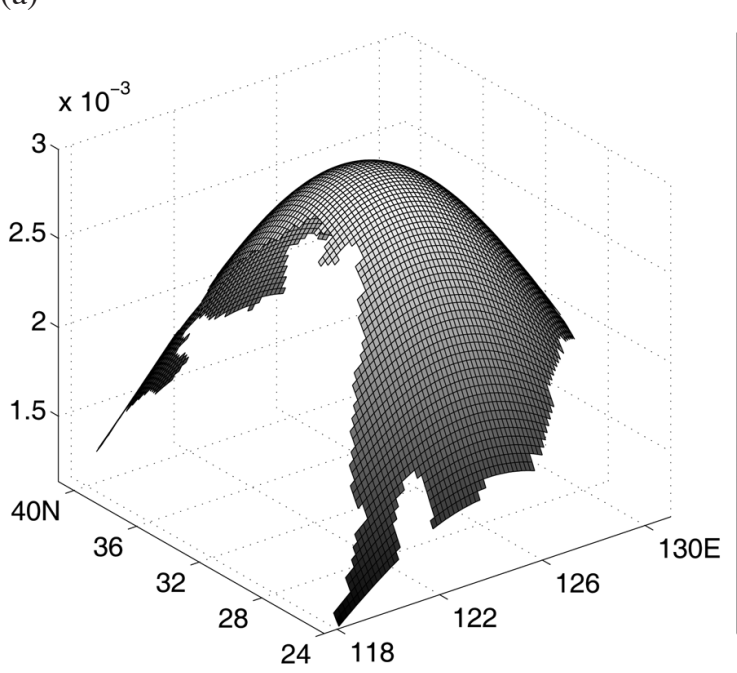

(b)

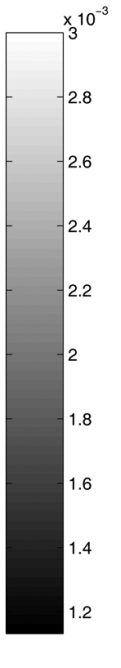

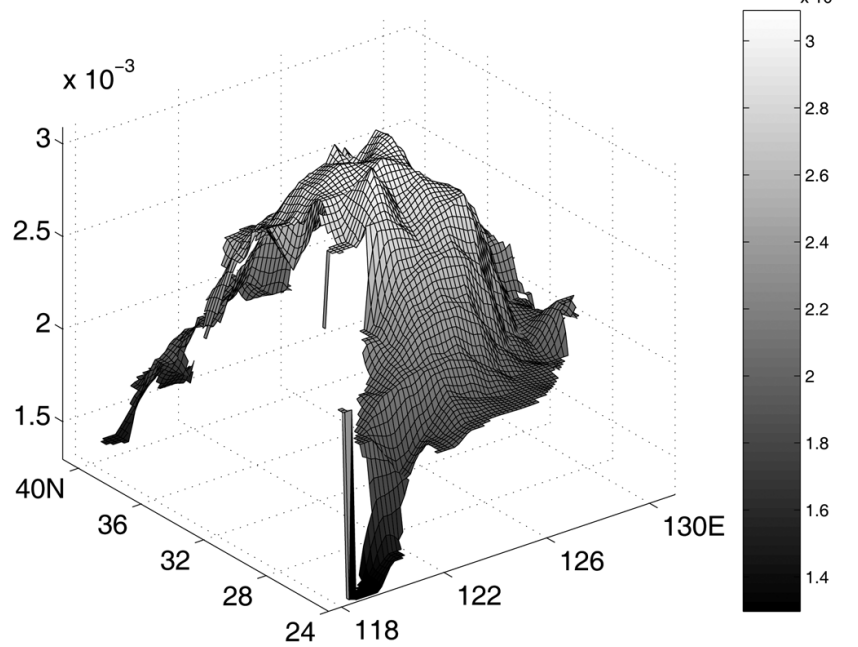

Fig. 8. Same as Fig. 4, but for case 5. 
(a)

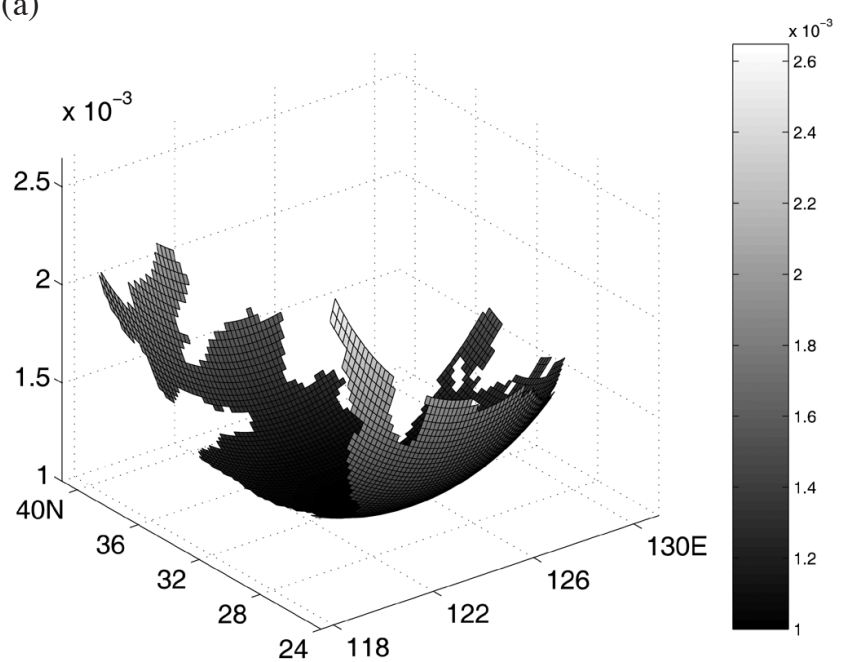

(b)

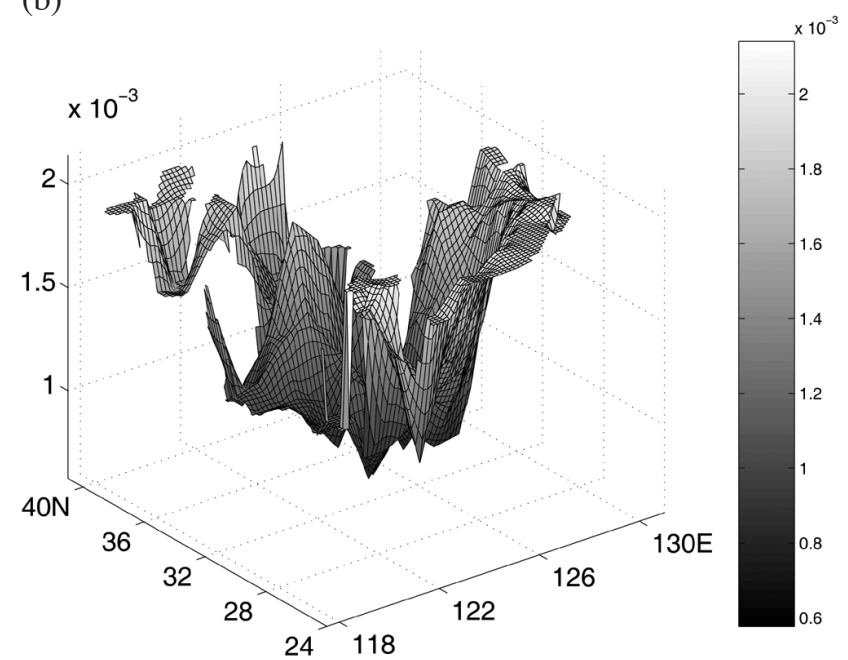

Fig. 9. Same as Fig. 4, but for case 6.

model. The following details can be discovered. For surfaces of different type, the inversion of conical surface is better than that of revolution parabolic surface and the inversion of revolution parabolic surface is better than that of revolution normal surface. The most probable reason is that the complexity of these surfaces is different. The more complicate the prescribed distributions are, the worse the inversion results will become. For the same type of surfaces, the inversion of the upward convex surfaces is better than that of the downward ones. Maybe it is because the upward convex surface distributions are more reasonable to adapt to the ocean topography of BYECS. According to the analysis above, we can conclude that the inversion of prescribed BFC with adjoint method has obtained satisfying results, and the complicated BFC distributions can be inverted reasonably by the method in this paper.

\subsection{Practical Experiments}

A practical experiment (PE1) is carried out to simulate the $\mathrm{M}_{2}$ tide in BYECS by optimizing the spatially varying BFC. Both the position and the value of $\mathrm{T} / \mathrm{P}$ data are used in PE1, which is different from the twin experiments. Data of 69 tidal gauge stations (Fig. 2) are used as an independent verification of the model fidelity. $K_{c}$ and the initial value of BFC are also taken as $10^{4}$ and 0.002 , respectively, in PE1. Therefore, according to the conclusion of the twin experiments, the optimization is terminated at the 150th step. For the sake of comparison, we choose another two traditional methods to deal with the BFC. In the first method, the BFC are taken as a constant (0.0018) in BYECS (PE2). In the second one, the BFC are the same as that of Zhao et al. (1993) (PE3). The absolute mean differences between T/P data and simulated values of 3 practical experiments are exhibited in Table 3.
From Table 3, one can find that, by optimizing the spatially varying independent BFC, the results of PE1 are better than those of PE2 and PE3. It indicates that the spatially varying $\mathrm{BFC}$ is more reasonable than the traditional methods. The bottom friction is determined by the ocean topography, so it would not be enough to figure out the complicated bottom friction clearly by the traditional methods while it can be done by the spatially varying BFC. In Fig. 10, the BFC spatial distribution of BYECS obtained from PE1 are plotted. The average water depth of the Bohai Sea is $19 \mathrm{~m}$ and the average BFC is $1.06 \times 10^{-3}$, while $39 \mathrm{~m}$ and 0.92 $\times 10^{-3}$ for the North Yellow Sea, $48 \mathrm{~m}$ and $0.83 \times 10^{-3}$ for the South Yellow Sea, $93 \mathrm{~m}$ and $2.04 \times 10^{-3}$ for the Korean Strait and the Strait of Tsushima, and $360 \mathrm{~m}$ and $2.15 \times 10^{-3}$ for the East China Sea. In detail, the Yellow Sea is divided into two parts by the Shandong Peninsula. One can find that the BFC along the coast are larger than those off the coast in the Bohai Sea, the North Yellow Sea, the South Yellow Sea and the East China Sea individually, which means that the $\mathrm{BFC}$ in shallow water are larger than those in deep water. Additionally, The BFC in the East China Sea are larger than those in the other areas for most of the grid points. Figure 11 gives the cotidal chart of $\mathrm{M}_{2}$ tide in BYECS which is obtained from PE1. There are three amphidromic points in BYECS: one in the Bohai Sea and two in the Yellow Sea. Near the Yellow River Mouth, the amphidromes appear as degenerated systems and Fang (1986) suggested that it was determined by the change of the coastline of the Yellow River Delta. The amphidromic point in the Bohai Sea is very close to the coast near Qinhuangdao of China. In the Yellow Sea, the amphidromic points are located northeast of Chengshantou and Southeast of Qingdao of China. The position deduced from this study is consistent with that of Fang et al. (2004) where the empirical cotidal charts of BYECS are obtained from 10 years of $\mathrm{T} / \mathrm{P}$ altimetry. In 
Table 3. The absolute mean differences of amplitude and phase-lag of water elevation between simulated values and observed data in practical experiments.

\begin{tabular}{ccccc}
\hline \multirow{2}{*}{ EXP. } & \multicolumn{2}{c}{ Average error of T/P data } & \multicolumn{2}{c}{ Average error of tidal gauges data } \\
\cline { 2 - 5 } & Amplitude $(\mathbf{c m})$ & Phase-lag $\left(^{\circ}\right)$ & Amplitude $(\mathbf{c m})$ & Phase-lag $\left(^{\circ}\right)$ \\
\hline PE1 & 5.4 & 5.3 & 7.7 & 8.0 \\
PE2 & 7.7 & 6.1 & 10.1 & 7.7 \\
PE3 & 7.3 & 6.3 & 9.8 & 8.0 \\
\hline
\end{tabular}

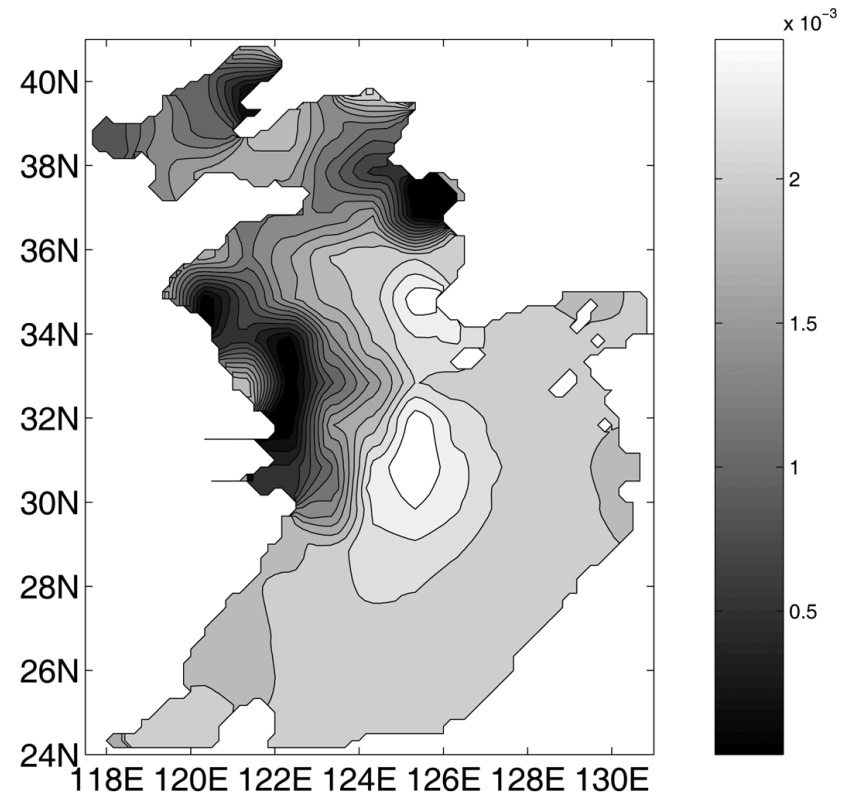

Fig. 10. The BFC distribution of BYECS obtained from PE1.

some studies, the amphidromic point near Qinhuangdao did not appear (Choi 1989; Yanagi and Inoue 1994). The selection of BFC may be responsible for this phenomenon (Guo and Yanagi 1998). In this paper, the BFC is supposed to be spatially varying and consequently the simulation results are reasonable.

\section{SUMMARY}

In this paper, the spatially varying BFC is studied by carrying out twin and practical experiments. The $\mathrm{M}_{2}$ tide in BYECS is simulated by assimilating the T/P altimetry data with the adjoint method. In this paper, the BFC at some grid points are chosen as the independent $\mathrm{BFC}$ and the $\mathrm{BFC}$ at the other grid points can be obtained through linear interpolation with these independent BFC. The independent $\mathrm{BFC}$ are selected uniformly over each $1^{\circ} \times 1^{\circ}$ area and 120 independent BFC are obtained. The prescribed BFC distri-

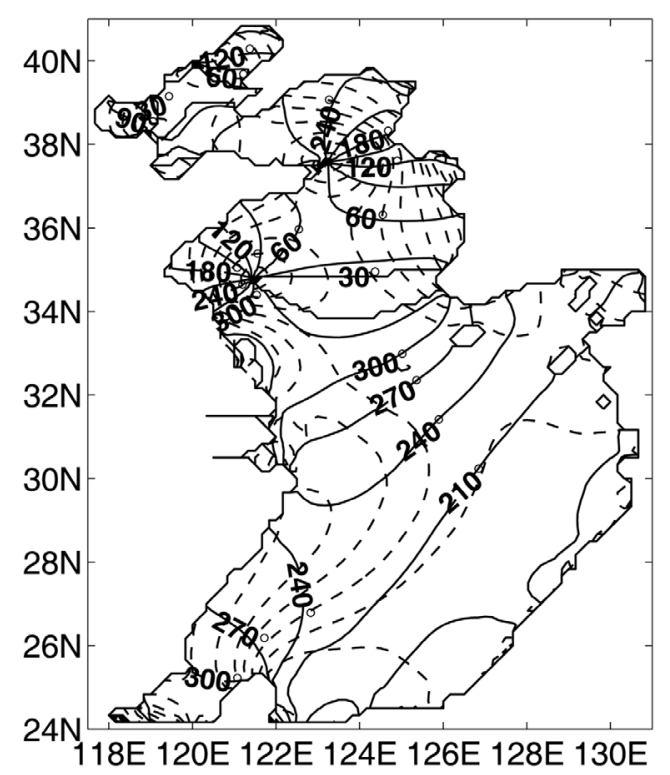

Fig. 11. The cotidal charts of $\mathrm{M}_{2}$ tide obtained from PE1. Solid line: phase-lag (in deg), dashed line: amplitude (in m).

butions are all successfully inverted, which demonstrates the strong ability of the adjoint model and the reasonability of the spatially BFC. Comparing with PE2 and PE3 which employ traditional BFC methods, the simulation precision of PE1 has been highly increased by optimizing the spatially varying independent BFC. Three amphidromic points and a degenerate one appear in the cotidal charts of $\mathrm{M}_{2}$ tide and the results coincide with the observed $\mathrm{M}_{2}$ tide in BYECS fairly well. The BFC distribution obtained from PE1 indicates that, the BFC along the coast are larger than those off the coast in the Bohai Sea, the North Yellow Sea, the South Yellow Sea and the East China Sea individually. Additionally, The BFC in the East China Sea are larger than those in the other areas for most of the grid points. The future work will discuss the influence of spatially varying BFC on tidal dissipation.

Acknowledgements We deeply thank the reviewers and editors for their constructive criticism of an early version 
of the manuscript. Partial support for this research was provided by the State Ministry of Science and Technology of China through grant 2007AA09Z118, the National Basic Research Program of China through grant 2005CB422308, and the Specialized Research Fund for the Doctoral Program of Higher Education through grant 20050423007. The TOPEX/POSEIDON data are provided by PODAAC of JPL.

\section{REFERENCES}

Anderson, O. B., 1995: Global ocean tides from ERS-1 and TOPEX/POSEIDON altimetry. J. Geophys. Res., 100, 25249-25259.

Choi, B. H., 1989: A fine-grid three-dimensional $\mathrm{M}_{2}$ tidal model of the East China Sea. In: Davies, A. M. (Ed.), Modeling Marine Systems, Boca Raton, Florida, 167185.

Cressman, G. P., 1959: An operational objective analysis system. Mon. Weather Rev., 87, 367-374.

Das, S. K., and R. W. Lardner, 1991: On the estimation of parameters of hydraulic models by assimilation of periodic tidal data. J. Geophys. Res., 96, 15187-15196.

Das, S. K., and R. W. Lardner, 1992: Variational parameter estimation for a two dimensional numerical tidal model. Int. J. Numer. Methods Fluids, 15, 313-327.

Egbert, G. D., A. Bennett, and M. Foreman, 1994: TOPEX/ Poseidon tides estimated using a global inverse model. J. Geophys. Res., 99, 24821-24852.

Egbert, G. D., R. D. Ray, and B. G. Bills, 2004: Numerical modeling of the global semidiurnal tide in the present day and in the last glacial maximum. J. Geophys. Res., 109, C03003.

Fang, G. H., 1986: Tide and tidal current charts for the marginal seas adjacent to China. J. Oceanol. Limnol., 4, 1-16.

Fang, G. H., Y. G. Wang, Z. X. Wei, B. H. Choi, X. Y. Wang, and J. Wang, 2004: Empirical cotidal charts of the Bohai, Yellow, and East China Seas from 10 years of TOPEX/Poseidon altimetry. J. Geophys. Res., 109, C11006.

Guo, X. Y., and T. Yanagi, 1998: Three-dimensional structure of tidal current in the East China Sea and the Yellow Sea. J. Oceanogr., 54, 651-668.

Heemink, A. W., E. E. A. Mouthaan, M. R. T. Roest, E. A. H. Vollebregt, K. B. Robaczewska, and M. Verlaan, 2002: Inverse 3D shallow water flow modelling of the continental shelf. Cont. Shelf Res., 22, 465-484.

He, Y. J., X. Q. Lu, Z. F. Qiu, and J. P. Zhao, 2004: Shallow water tidal constituents in the Bohai Sea and the Yellow Sea from a numerical adjoint model with TOPEX/ POSEIDON altimeter data. Cont. Shelf Res., 24, 15211529.

Kang, S. K., S. R. Lee, and H. J. Lie, 1998: Fine grid tidal modeling of the Yellow and East China Seas. Cont. Shelf Res., 18, 739-772.

Kantha, L., 1995: Barotropic tides in the global oceans from a nonlinear tidal model assimilating altimetric tides, I, Model description and results. J. Geophys. Res., 100, 25283-25308.

Lefevre, F., C. Le Provost, and F. H. Lyard, 2000: How can we improve a global ocean tide model at a regional scale? A test on the Yellow Sea and the East China Sea. J. Geophys. Res., 105, 8707-87257.

Le Provost, C., M. L. Genco, F. H. Lyard, P. Vincent, and P. Canceil, 1994: Spectroscopy of the world ocean tides from a finite element hydrodynamic model. J. Geophys. Res., 99, 24777-24797.

Lu, X. Q., and J. C. Zhang, 2006: Numerical study on spatially varying bottom friction coefficient of a $2 \mathrm{D}$ tidal model with adjoint method. Cont. Shelf Res., 26, 19051923.

Mazzega, P., and M. Berge, 1994: Ocean tides in the Asian semi-enclosed seas from TOPEX/POSEIDON. J. Geophys. Res., 99, 24867-24881.

Teague, W. J., P. Pistek, G. A. Jacobs, and H. T. Perkins, 2000: Evaluation of tides from TOPEX/Poseidon in the Bohai and Yellow Seas. J. Atmos. Ocean. Technol., 17, 679-687.

Ullman, D. S., and R. E. Wilson, 1998: Model parameter estimation from data assimilation modelling: Temporal and spatial variability of the bottom drag coefficient. $J$. Geophys. Res., 103, 5531-5549.

Munk, W. H., 1997: Once again: Once again-tidal friction. Prog. Oceanogr., 40, 7-35.

Yanagi, T., and K. Inoue, 1994: Tide and tidal current in the Yellow/East China Seas. La mer, 32, 153-165.

Yangi, T., A. Morimoto, and K. Ichikawa, 1997: Co-tidal and co-range charts for the East China Sea and the Yellow Sea derived from satellite altimetric data. $J$. Oceanogr., 53, 303-309.

Zhao, B. R., G. H. Fang, and D. M. Cao, 1993: Numerical modeling on the tides and tidal currents in the Eastern China Sea. Yellow Sea Res., 5, 41-61. 